\title{
Polarity-directed analog electro-optic switching in a low-polarization chiral smectic liquid crystal with positive dielectric anisotropy
}

\author{
Jan P. F. Lagerwall, * Alexander A. Kane, and Noel A. Clark \\ Department of Physics, University of Colorado, Boulder, Colorado 80309, USA \\ David M. Walba \\ Department of Chemistry and Biochemistry, University of Colorado, Boulder, Colorado 80309, USA \\ (Received 2 February 2004; published 21 September 2004)
}

\begin{abstract}
We describe an analog electro-optic (EO) switching mechanism occurring in thin cells filled with a lowpolarization ferroelectric liquid crystal mixture with positive dielectric anisotropy. The mixture is composed of an achiral nonpolar smectic- $C(\mathrm{Sm}-C)$ host doped with a small amount of a commercially available unichiral compound. The switching mechanism provides analog EO behavior, and thus could be attractive for information display applications. The process is polarization-driven for weak fields, while for higher field strength the dielectric coupling dominates the process.
\end{abstract}

DOI: 10.1103/PhysRevE.70.031703

PACS number(s): 77.84.Nh, 77.80.Fm, 42.79.Kr

\section{INTRODUCTION}

Chirality in liquid crystals has many striking consequences, from helical superstructures and huge optical activity to the spontaneous appearance of mesoscopic electric polarization. The latter appears in a smectic- $C(\mathrm{Sm}-C)$ phase which is made chiral $\left(\mathrm{Sm}-C^{*}\right)$ [1], either by using a chiral substance which forms the Sm- $C^{*}$ phase or by doping an achiral Sm- $C$ host with a chiral dopant $[2,3]$. The Sm- $C$ phase is characterized by a layered structure where the director $\mathbf{n}$ (the average molecule long axis direction) is tilted by a nonzero angle $\theta$ (generally temperature dependent) with respect to the layer normal $\mathbf{z}$. In a nonchiral smectic- $C$ phase a spontaneous polarization is forbidden by the symmetry of the phase. Hence the response to an electric field $\mathbf{E}$ cannot be polar (sensitive to the sign of the field). The same holds for a perfect racemate, i.e., an exact 1:1 mixture of $(S)$ - and $(R)$-enantiomers of the same chiral compound. But a nonpolar (dielectric) response to an electric field applied along the layers of a Sm- $C$ sample can be induced due to the dielectric anisotropy of the liquid crystal. This switching mechanism, in which $\mathbf{n}$ reorients on the smectic- $C$ tilt cone (the cone around $\mathbf{z}$ with opening angle $2 \theta$ that contains all allowed orientations of $\mathbf{n}$ ), has been extensively investigated by Pelzl and co-workers. They showed that the origin of the dielectric switching in a nonchiral Sm- $C$ phase, just like in the nematic phase, basically is an instability of the Frederiks transition type [4-7].

The transformation from nonpolar to polar behavior of a Sm- $C$ phase which is made chiral through doping makes this phase an excellent basis for a chirality detection device. Here we report on the doping of a nonchiral $\mathrm{Sm}-C$ host with a small amount of a chiral nonliquid crystalline compound derived from a pharmaceutical substance, giving the mixture a

\footnotetext{
*Present address: Institute of Physical Chemistry, University of Stuttgart, Pfaffenwaldring 55, D-70569 Stuttgart, Germany. Email address: jan.lagerwall@ipc.uni-stuttgart.de
}

spontaneous polarization $\mathbf{P}$ which despite its very small magnitude radically changes the electro-optic switching behavior. We analyze the complex resulting switching mechanism and show that it has quite attractive properties for use in electrooptic devices, since it offers fast analog response and good contrast. Since the discovery of the polar nature of the Sm- $C^{*}$ phase, several mechanisms for electro-optic switching utilizing the coupling between $\mathbf{P}$ (which is directed perpendicular to $\mathbf{n}$ and $\mathbf{z}$ ) and an applied electric field have been proposed. Initially, the discovery that bistable ferroelectric switching could be obtained [8]-hence Sm- $C^{*}$ liquid crystals are generally called ferroelectric liquid crystals (FLCs) — created a great interest in bistable modes. In recent years much interest has been on analog $\mathrm{Sm}-C^{*}$ switching modes as these give inherent gray scale capabilities. One of the most intense discussions in this area has been focused on FLC materials with very high polarization [9-12]. The analog mode described in the present paper occurs at the opposite extreme, in FLCs with very low polarization. The mechanism is to a large extent a dielectric one. The role of the polarization is mainly to remove the Frederiks threshold and to introduce a sensitivity to the sign of the applied field. Because the required polarization is very small, the same switching mechanism may be encountered also in $(S)+(R)$-FLCs with positive dielectric anisotropy and chiral components that are only slightly enantiomerically enriched.

\section{EXPERIMENT}

The nonchiral Sm- $C$ host used for this study was a mixture, all five components of which have phenylpyrimidine cores, prepared by Displaytech Inc. and code-named 8055. It exhibits the nontilted smectic- $A(\mathrm{Sm}-A)$ and the nematic $(\mathrm{N})$ phase in addition to $\mathrm{Sm}-C$. The mixture has positive dielectric anisotropy $(\Delta \epsilon>0)$ in the $\mathrm{Sm}-A$ phase and weak biaxiality in $\mathrm{Sm}-C$, such that the $\mathrm{Sm}-C$ tilt plane aligns parallel to an applied ac electric field. The saturated $\theta$ value in the Sm- $C$ phase was about $20^{\circ}$. The 8055 host was doped with $2 \%$ (by weight) of the chiral non-liquid-crystalline com- 


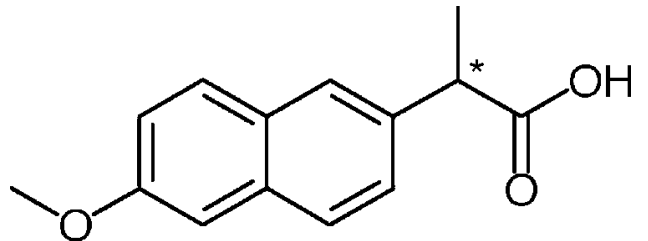

FIG. 1. The structure of the crystalline chiral dopant compound [(+)-2-(6-Methoxy-naphthalen-2-yl)-propionic acid].

pound (+)-2-(6-Methoxy-naphthalen-2-yl)-propionic acid (compound 1), commercially available from Aldrich Chemical Co., Inc. The structure of compound $\mathbf{1}$ (melting point $250{ }^{\circ} \mathrm{C}$ ) is given in Fig. 1. The phase sequences of host 8055 with and without chiral dopant are listed in Table I.

The mixtures were filled by capillary action in the isotropic phase into $1.5 \mu \mathrm{m}$ thick sample cells prepared at the cell assembly line of MC2, Chalmers (Göteborg, Sweden). The cells were equipped with transparent ITO electrodes and antiparallel buffed polyimide coatings producing planar alignment. Electro-optic experiments were carried out using a photodiode mounted on a Nikon Optiphot Pol microscope with a $40 \times$ objective, ensuring that the alignment was homogeneous throughout the investigated sample area. The light source was a $633 \mathrm{~nm} \mathrm{He}-\mathrm{Ne}$ laser.

\section{RESULTS AND DISCUSSION}

\section{A. The nonchiral Sm-C host}

Before studying the chirally doped mixture, the 8055 host was studied on its own. The $\mathrm{Sm}-C$ phase texture exhibited zig zag defects showing that a chevron structure had developed as a result of the layer shrinkage below the Sm- $A-C$ transition [13]. With antiparallel buffing one might expect layer tilting rather than chevron formation in the Sm-C phase. However, the pretilt within the active area was small in the cells used and this promotes the chevron structure [14]. The texture in the electrode area was, apart from the defects, uniform with an extinction direction which was identical to that of the Sm- $A$ phase, cf. Fig. 2(a). No separation into domains with optical tilt of $+\theta$ and $-\theta$, as one might intuitively expect with a $\mathrm{Sm}-C$ phase between planaraligning surfaces, occurred here. Outside the electrode area, on the other hand, where the alignment layer was applied directly on glass, such domains did form.

When a voltage (dc or ac) above the Frederiks threshold was applied a domain pattern formed in the electrode area

TABLE I. Phase sequences (on heating) of the liquid crystal mixtures studied. Temperatures in ${ }^{\circ} \mathrm{C}$.

\begin{tabular}{lcccc}
\hline \hline \multicolumn{1}{c}{$\mathrm{Cr}$} & $\mathrm{Sm}-C$ or $C^{*}$ & $\mathrm{Sm}-A$ or $A^{*}$ & $N$ or $N^{*}$ & Iso \\
\hline $\begin{array}{l}8055 \\
\text { (nonchiral) }\end{array}$ & $<20$ & $\sim 49$ & $\sim 61$ & $\sim 70$ \\
$\begin{array}{l}8055 \\
\mathbf{1}\end{array}$ & $<20$ & $\sim 42$ & $\sim 49$ & $\sim 66$ \\
(chiral) & & & & \\
\hline \hline
\end{tabular}
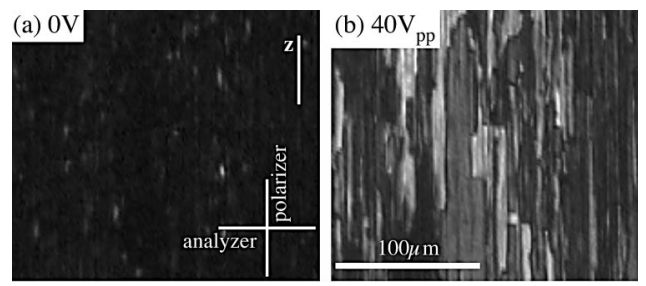

FIG. 2. The achiral sample exhibited a Sm- $C$ texture which in the absence of field (a) was uniform with the effective optic axis along the layer normal $\mathbf{z}$ (parallell to cell buffing direction). The application of a $65 \mathrm{~Hz}$ square wave voltage above a threshold level $\left(\approx 34 \mathrm{~V}_{p p}\right)$ generated a domain texture $(\mathrm{b})$.

[Fig. 2(b)]. In the domains the effective optic axis was tilted away from $\mathbf{z}$, the tilt magnitude the same but the direction opposite between the two domain types. The pattern was insensitive to the voltage sign and it reformed with exactly the same domains if the voltage was switched off and then on again. In order to measure the magnitude of the induced optical tilt angle $\theta_{\text {opt }}$ (the angle between the effective optic axis and the cell plane projection of $\mathbf{z}$ ) a square wave ac voltage with increasing amplitude was applied. The sample was then rotated to the extinction positions of the two domain types at each voltage level. On increasing the voltage from the Frederiks threshold value $\left(\sim 34 \mathrm{~V}_{p p} ; p p=\right.$ peak-topeak), below which $\theta_{\text {opt }}=0, \theta_{\text {opt }}$ first increased sligthtly in magnitude but then started decreasing with increasing voltage, cf. Fig. 3.

Although we have no x-ray data on the smectic layer thickness as a function of temperature for host 8055, the usual situation in Sm- $A-C$ materials is that the layer shrinkage below the tilting transition is smaller than what the magnitude of $\theta$ would suggest, hence the chevron inclination $\delta$ in the sample is assumed to be somewhat smaller than $\theta$. The situation is illustrated in Fig. 4, where the left-most column depicts the state in the absence of field. For the sake of generality we allow an asymmetry in the position of the chevron interface, placing it slightly off center. As is clear from the in-layer view (a), the combination of planar surface anchoring with small pretilt and chevron geometry means that the director is close to the top or bottom of the smectic$C$ tilt cone at each surface.

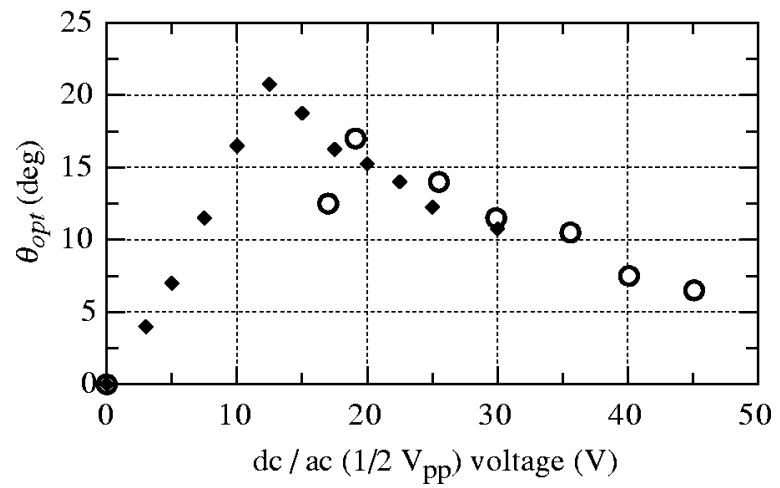

FIG. 3. The optical tilt angle $\theta_{\text {opt }}$ in the achiral host 8055 (empty circles) and in the chirally doped mixture (filled diamonds), as a function of applied voltage over $1.5 \mu \mathrm{m}$ thick samples. 


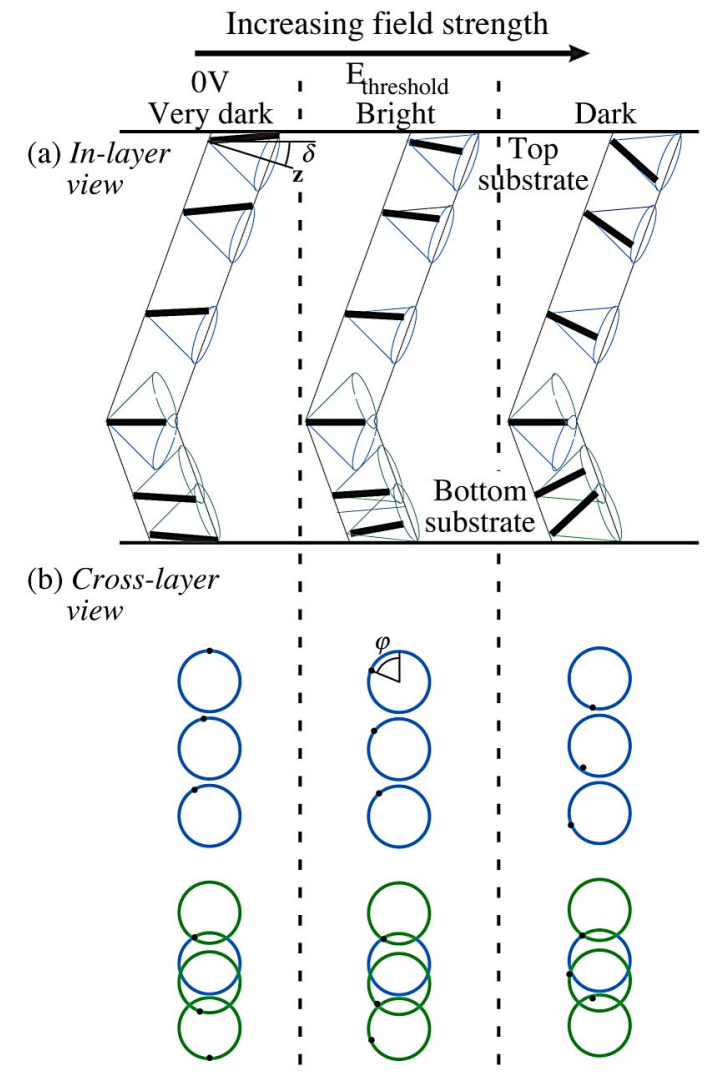

FIG. 4. An illustration of the chevron geometry in the nonchiral sample and the changes in director orientation [thick lines in (a) and black dots in (b)] induced by the dielectric coupling to an applied electric field, directed vertically.

An electric field applied from substrate to substrate couples to the positive dielectric anisotropy of the liquid crystal, promoting an alignment of $\mathbf{n}$ perpendicular to the cell plane. Since $\mathbf{n}$ is restricted to the surface of the tilt cone (we do not consider field strengths high enough to distort the smectic layer structure) any field-induced change must take place via changes in the azimuthal angle $\varphi$, i.e., director rotation on the cone surface. Small fields leave the sample unaffected: in case the pretilt is zero the electric field exhibits no torque on the director in its perfectly planar zero-field state, whereas with a small pretilt the states in the immediate vicinity of the zero-field state will actually be more planar than the initial state. The process of director reorientation thus starts with a Frederiks transition once the voltage reaches a certain threshold level [4,5]. The director then switches along the cone surface to the second state depicted in Fig. 4.

The magnitude of the effective optical tilt $\theta_{\text {opt }}$ can be deduced for the case of a uniform director structure from substrate to substrate from the sketches in Fig. 5. Part (a) depicts the bookshelf case, i.e., corresponding to a chevron inclination angle of $\delta=0$. Geometrical considerations, using the variables defined in the figure, give

$$
\tan \theta_{\mathrm{opt}}=b / z=\frac{r \sin \varphi}{r / \tan \theta}=\sin \varphi \tan \theta .
$$
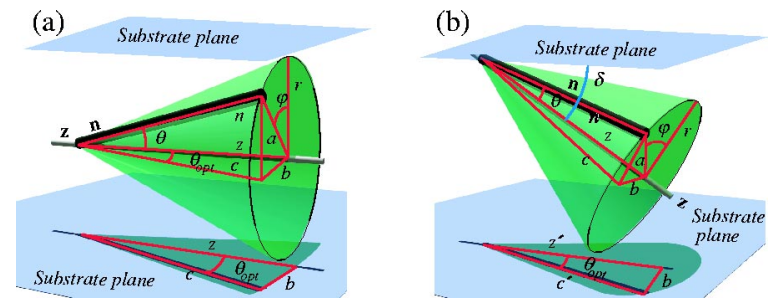

FIG. 5. Sketches illustrating the geometrical relations between the effective optical tilt angle and the director tilt angle in the (a) bookshelf and (b) chevron geometry cases.

In the chevron case, the tilt of the layer normal $\mathbf{z}$ leads to a shortening of its projection to a length $z^{\prime}$, but the perpendicular distance between the layer normal and the end of the director projection, $b$, remains unchanged. We can thus use the expression for the bookshelf case also for the chevron geometry, multiplied by a factor reflecting the layer normal tilt,

$$
\tan \theta_{\mathrm{opt}}=b / z^{\prime}=\frac{r \sin \varphi}{(r / \tan \theta) \cos \delta}=\frac{\sin \varphi \tan \theta}{\cos \delta} .
$$

Thus, for values $\theta=20^{\circ}$ and $\delta=20^{\circ}$, which are good approximations for the mixtures studied in this work, the chevron geometry leads to an increase of the maximum optical tilt angle (obtained for $\varphi=90^{\circ}$ ) of about 1 degree. However, the samples studied exhibit a more or less strong twist in the director structure (see below), generally leading to a small decrease of $\theta_{\text {opt }}$ which may compensate this effect.

Because the chevron geometry renders the cone tilted with respect to the cell plane, $\varphi$ is in the first field-induced state less than $90^{\circ}$, hence the sample, viewed along the substrate normal, does not exhibit its maximum optical tilt angle. Therefore, the measured value of $\theta_{\mathrm{opt}}$ in Fig. 3 continued to increase slightly for voltages just above the threshold level. The decrease started first when the voltage was high enough to push $\mathbf{n}$ to the other cone half $\left(\varphi>90^{\circ}\right)$, moving the cell plane projection of $\mathbf{n}$ closer to the projection of $\mathbf{z}$, as in the right column of Fig. 4.

At the chevron interface the director is locked in one of two orientations, both parallel with the substrate plane [15]. Therefore, the director here (in the following we denote this $\mathbf{n}_{C I}$ ) was not affected by the electric field and there was in general a twisted structure mediating $\mathbf{n}_{C I}$ with $\mathbf{n}$ at the cell surface. The side of the cone along which $\mathbf{n}$ moved during switching was set by $\mathbf{n}_{C I}$. Since $\mathbf{n}_{C I}$ can never be switched in the achiral sample the domain texture formed during switching always looked the same (as long as the sample was not heated out of the $\mathrm{Sm}-C$ phase).

\section{B. The chirally doped mixture}

Going to the chiral sample, the two most important differences are the appearance of a nonzero spontaneous polarization $\mathbf{P}$ and a preference of one twisting sense over the other. The first effect of the polarization that we must take into account is the so-called surface electroclinic effect [16]. As a surface in contact with an FLC acts like an electric field directed into or out of the sample [17] $\mathbf{P}$ will tend to be 


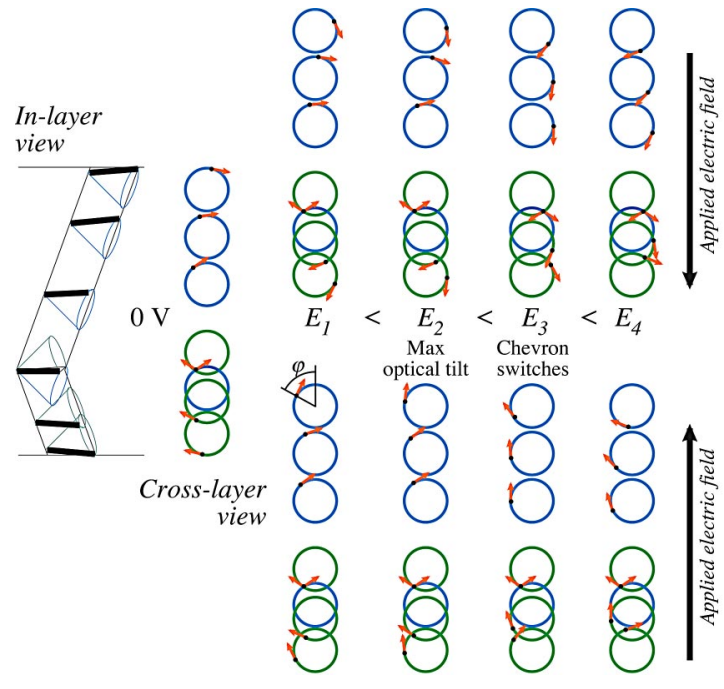

FIG. 6. An illustration of the chevron geometry in the chiral sample and the changes in director orientation (thick lines in the in-layer view, black dots in the cross-layer view) induced by the combination of dielectric and polar coupling to an applied electric field. The two rows on the right part display the nonequal responses for fields directed up and down, respectively, starting from the same zero-field state (left). The arrows represent $\mathbf{P}$.

directed into or out of the surfaces. Because $\mathbf{P}$ is directed along $\mathbf{n} \times \mathbf{z}$ this means that a surface director at the top or bottom of the cone will experience a torque trying to reorient $\mathbf{n}$ along the cone surface in one specific sense. Since $\mathbf{P}$ was very small in the doped host 8055 mixture and as the low pretilt pushed $\mathbf{n}$ towards the top/bottom of the cone, the effect was in our case small but nevertheless had visible consequences. The resulting zero-field director structure is schematically drawn to the left in Fig. 6.

As in the case of the undoped sample, the extinction direction in $\mathrm{Sm}-C^{*}$ in the absence of field was parallel to $z$ in the electrode area. The sample texture was essentially uniform, apart from a few zig zag defects which revealed that the liquid crystal adopted a chevron structure. Domains corresponding to optical tilt $+\theta$ and $-\theta$ (now equivalent to polarization up and down), respectively, were again seen only outside the electrode area.

In contrast to the case of the undoped sample, application of a dc voltage invoked the same response across the whole electrode area. Furthermore, the direction of deviation of the effective optic axis now depended on the sign of the voltage, showing that the chirally doped mixture was polar. However, the switching was not ferroelectric as the only stable state was the zero field state where the effective optic axis was aligned with the projection of $\mathbf{z}$. The torque induced by the polar coupling is given by $\mathbf{P} \times \mathbf{E}$. Since $\mathbf{P}$ at the surface was directed close to perpendicular to $\mathbf{E}$ in the zero-field state the polar torque on the near-surface director was close to maximum as soon as a voltage was applied to the cell. Therefore there was no threshold to distort the zero-field state, and $\theta_{\text {opt }}$ increased continuously and quasilinearly from zero as the voltage over the cell was increased from zero, cf. Fig. 3. After reaching a peak value of $\theta_{\text {opt }} \sim 21^{\circ}$ the tilt angle started to decrease with further increasing voltage, as in the achiral sample.
In the absence of field the texture, with the sample aligned for extinction, had a weak bluish tone indicating the presence of a light director twist from one substrate to the other, an effect of the different director orientations at the chevron interface and at the surfaces. The twist was present also in the achiral sample but it was now enhanced by the surface electroclinic effect to a magnitude giving visible consequences. For voltages up to $13 \mathrm{~V}$, i.e., the voltage which induced maximum $\theta_{\text {opt }}$, this color tone grew stronger for one sign of the voltage whereas it disappeared for the other. As the voltage was increased above this value the color disappeared almost instantly, suggesting that the twist was unwound at voltages stronger than that corresponding to maximum $\theta_{\text {opt }}$. As illustrated in Fig. 6, the combination of planar surface anchoring, small surface electroclinic effect, and chevron structure leads to a twist above or below the chevron interface, but an essentially uniform structure on the other side in the field-free state. Which side is more twisted, and which sense the twisting has, depends on how $\mathbf{n}_{C I}$ is oriented. Since the liquid crystal is now chiral, one twisting sense will be favored over the other and thus effectively only one orientation of $\mathbf{n}_{C I}$ is allowed at zero field. The chevron interface director is therefore uniformly aligned across the sample surface, in contrast to the achiral case. As is clear from the figure (field strengths $E_{1}$ and $E_{2}$ ), the chevron structure with $\mathbf{n}_{C I}$ fixed makes the positive and negative fieldinduced states quite different. Whereas one sign of the field keeps the sample essentially uniform from one substrate to the other, the other sign of the field generates a strongly twisted structure. This is why only one sign of the voltage generated the bluish dark state. Once the voltage reaches the level $E_{3}$ in the upper row, the twist is so strong that $\mathbf{n}_{C I}$ switches to the other allowed position in order to reduce the elastic energy, explaining why the color tone vanished at voltages above $13 \mathrm{~V}$. If the voltage was thereafter reduced to zero, $\mathbf{n}_{C I}$ switched back in order for the relaxed structure to re-adopt the natural twisting sense.

Due to the difference in director structures induced by positive and negative fields, we can expect some asymmetry in switching times. At field strength $E_{2}$ in the upper row of Fig. 6, the rather strong twist will tend to pull $\mathbf{n}$ back to its initial position, leading to a fast relaxation once the voltage decreases. In contrast, at a field of the opposite sign with strength $E_{2}$ - the lower row in the figure-the structure is only weakly twisted and we can expect a slower relaxation to the initial state. This is indeed what we found experimentally. In Fig. 7 we have plotted the switching time $\tau$ as a function of voltage, measured with a square driving waveform applied over the sample, aligned such that the weakly twisted $E_{2}$ state gave extinction. For all voltages below the threshold for switching of $\mathbf{n}_{C I}\left(E_{3} \approx 22 \mathrm{~V}_{p p}\right)$, the weak-tostrong-twist switching (rise time in the figure) was considerably slower than switching in the opposite direction. However, above $E_{3}$ the situation was reversed and, in addition, $\tau$ started to diverge. Both switched states now exhibited the same degree of twist, but not the same twisting sense. The state in the upper row of Fig. 6 has the natural twisting sense (same as at $E=0$ ) whereas the opposite state twists against the sense promoted by the chiral dopant. Therefore, the switching from the lower $\geqslant E_{3}$ state to the upper one was now faster than in the opposite direction. 


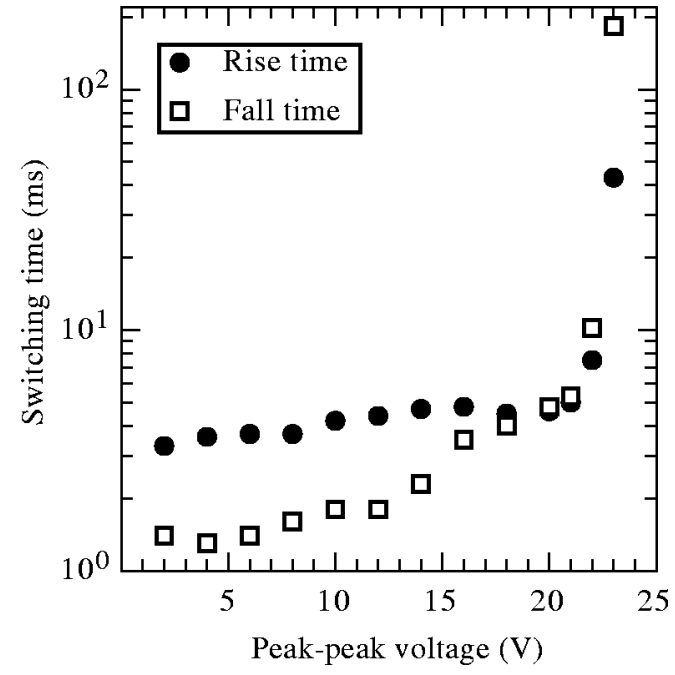

FIG. 7. Square wave switching time $\tau$. The frequency was lowered from $30 \mathrm{~Hz}$ at $2 \mathrm{~V}_{p p}$ to $1.5 \mathrm{~Hz}$ at $23 \mathrm{~V}_{p p}$ in order to allow complete relaxation between the sign changes of the driving waveform. The sample was aligned such that the rise time corresponded to switching from weak to strong twist, fall time the opposite direction, for voltages below $\sim 22 \mathrm{~V}_{p p}$. At higher voltages both states had about the same degree of twist. Note the logarithmic $y$ axis.

The slightly unusual situation with a switching time that increases with increasing field strength is a result of the polar and dielectric coupling mechanisms being comparable in strength, and of the way $\tau$ was measured. We measured the polar switching time, i.e., the response time to a sign change of a voltage of constant strength, a change which does not induce any dielectric switching. However, because the path from one polarly switched state to the other goes via director orientations that are more planar than the initial and end states, the dielectric coupling counteracts the polar switching. Because the dielectric energy term has a quadratic dependence on $\mathbf{E}$ while the polar coupling gives a linear energy term, the dielectric retardation becomes more important with increasing field strength, leading to an increase in $\tau$.

If a triangular ac voltage was applied the texture lost its homogeneity above a threshold level of $\sim 35 \mathrm{~V}_{p p}$, where a domain texture started to develop in parts of the sample, cf. Figs. 8(c) and 8(d). Within the domains the optical response to the variations in the driving voltage was very weak. If the sample was rotated it was possible to get good extinction in the domains, showing that the average effective optic axis in the domains was locked in one place, not parallel to $\mathbf{z}$. The director in the domains had thus apparently been switched to one end state, but thereafter the switching stopped. As the voltage was increased, more and more nonswitching domains were stabilized. If instead a square wave voltage was applied [Figs. 8(e) and 8(f)], the domains appeared more instantaneously and at a lower threshold. When the applied voltage reached $\sim 23 \mathrm{~V}_{p p}$, i.e., the level corresponding to maximum $\theta_{\mathrm{opt}}$ in the dc study (cf. Fig. 3) and divergence of switching times in Fig. 7, the uniformly switching texture transformed into a stable texture characterized by large domains with opposite optical tilt. On further increased voltage, the average transmission decreased continuously due to the reduction in magnitude of $\theta_{\text {opt }}$ [Fig. 8(f)].
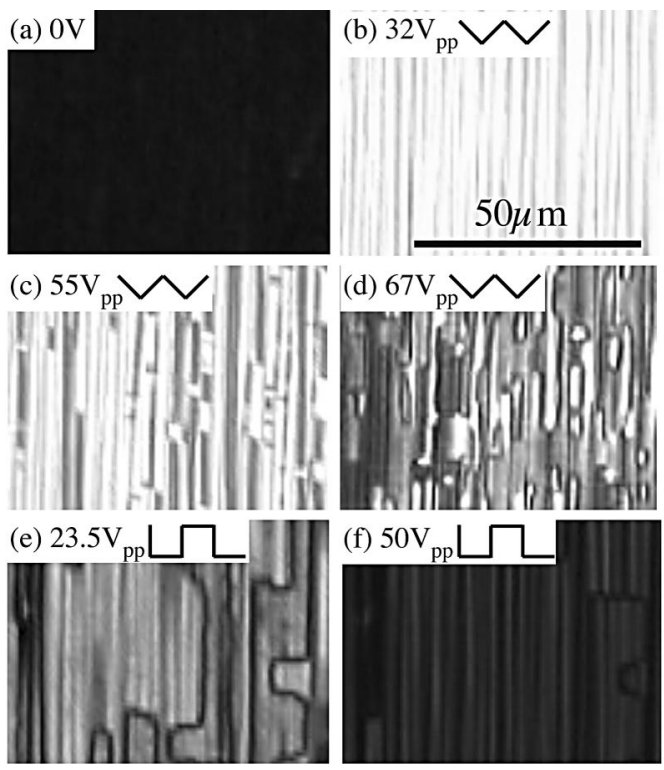

FIG. 8. Textures of the chirally doped sample at room temperature as the amplitude of an applied ac voltage $[65 \mathrm{~Hz}$, triangular waveform in (b)-(d), square wave in (e) and (f)] is increased. The optical transmission reached a maximum at $\sim 30 \mathrm{~V}_{p p}$ and $\sim 20 \mathrm{~V}_{p p}$, respectively, at which the texture was uniform for both waveforms. At higher voltages it broke up into a domain structure and the optical transmission decreased.

The reason for the domain formation is that at high voltage the coupling of the small polarization of this liquid crystal with the electric field is overpowered by the dielectric coupling. When the field is reversed, the polar coupling tries to move $\mathbf{n}$ laterally to the opposite side of the cone axis. However, far away from the chevron interface this lateral movement is now quite small, since the dielectric coupling will keep $\mathbf{n}$ down at the top half of the sample and up at the bottom. The twist is therefore almost as strong regardless of the position of $\mathbf{n}_{C I}$, rendering the driving force for switching it so weak that $\mathbf{n}_{C I}$ stays in the same place even when the voltage changes sign. With $\mathbf{n}_{C I}$ fixed and with $\mathbf{n}$ close to the surface oriented away from the surface and almost not moving, the effective optic axis when viewing the sample along the substrate normal reorients very little, hence the switching is in effect terminated at high voltages.

Dynamic electro-optic measurements were carried out using a triangular driving waveform with the sample aligned with $\mathbf{z}$ parallel to the polarizer, such that the optical transmission was minimum at $E=0$. Experiment results below, at and well above the threshold for domain formation are displayed in Fig. 9. For voltages up to $\sim 25 \mathrm{~V}_{p p}$ [Fig. 9(a)] the optical response (dashed curve) was semisinusoidal and essentially symmetric with respect to the sign of the applied voltage. The response exhibited a considerable phase lag with respect to the driving waveform (dotted curve). As expected for analog switching, no polarization peak was seen in the current response (fully drawn curve).

When the voltage reached the threshold for domain formation, an asymmetry between positive and negative voltages was generally introduced in the optical response and the intensity maxima split into two peaks, cf. Figs. 9(b) and 9(c). 

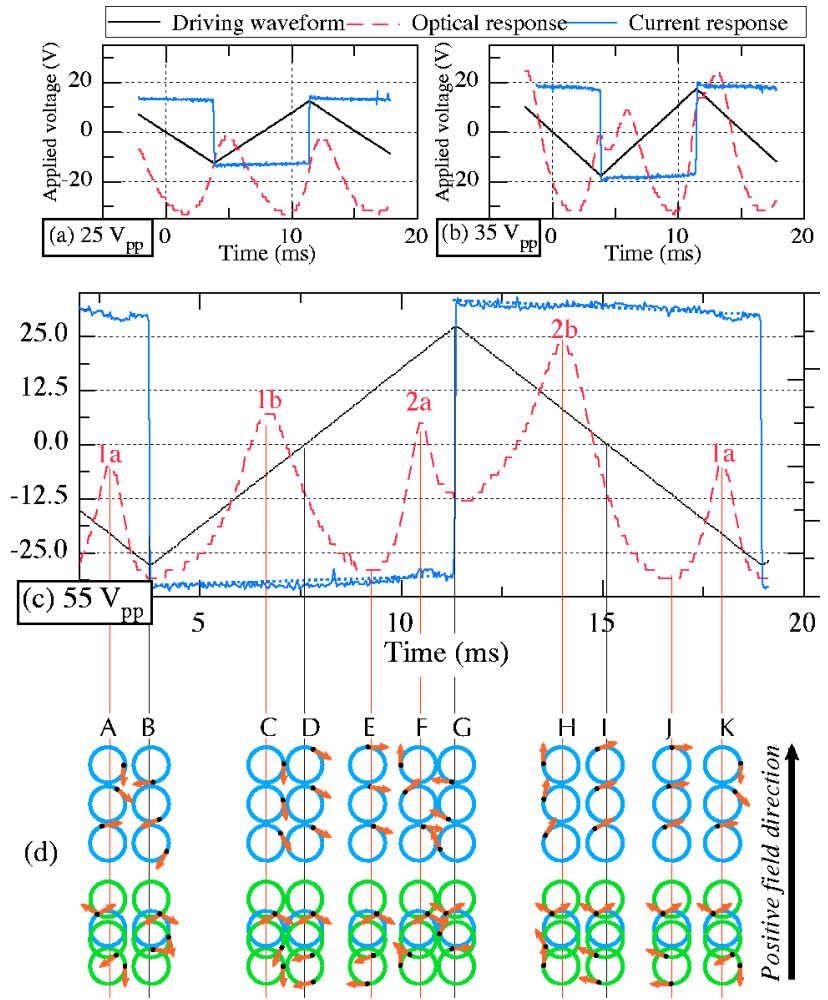

FIG. 9. Results from electro-optic measurements on the chirally doped mixture. The applied voltage (black curve) was (a) below, (b) at, and (c) well above the threshold for domain formation. The cartoon, with the tilt cone openings of the two chevron parts drawn in blue and green, respectively, the director with black dots and the polarization with red arrows, illustrates the different stages of the switching in case (c).

The first peak coincided with a very small polarization peak in the current response curve [best seen in (c) where it is separated from the border between positive and negative current response]. By integrating the peak obtained at $55 \mathrm{~V}_{p p}$ a polarization value of $\sim 4 \mathrm{nC} / \mathrm{cm}^{2}$ was obtained. However, it is questionable if this is a correct measure of the spontaneous polarization, since the process observed was not normal ferroelectric switching. The peak even had the wrong sign, decreasing instead of adding to the current response. This indicates a different nature of the peak, to be discussed below.

To understand the complex electro-optic and current response we analyze the $55 \mathrm{~V}_{p p}$ response [Fig. 9(c)], where domain formation was strong but a large part of the sample still switched. A texture photograph obtained during switching with this voltage is shown in Fig. 8(c) and a model for the switching mechanism is drawn at the bottom of Fig. 9, below the corresponding response curves. We take the transmission minimum occurring shortly after the driving waveform crossed zero with positive slope as the starting point for our analysis. In the cartoon this is state $\mathrm{E}$, basically equivalent to the zero-field state in the dc case depicted in Fig. 6. When the voltage increased, the director far away from the chevron moved towards the side of the cone where $\mathbf{P}$ aligned with the field. As a result, a tilt of the effective optic axis away from $\mathbf{z}$ developed, leading to an increase in light trans- mission. However, $\mathbf{n}_{C I}$ was locked at the other side of the cone, so the structure that arose (column F) exhibited a twist from one substrate to the other. This reduced the transmission slightly and therefore the transmission maximum at state F (labeled 2a) was not the global maximum. Right after 2a a threshold voltage was reached where the twisted state was no longer stable and $\mathbf{n}_{C I}$ switched. As the voltage increased further the dielectric coupling dominated over the polar coupling, driving $\mathbf{n}$ towards the bottom/top of the cone. The final structure before the voltage decreased again, column G, therefore exhibited a small effective optical tilt angle and we saw a minimum in optical transmission. This was not seen for voltages up to $25 \mathrm{~V}_{p p}$, since the dielectric torque was then not strong enough to drive $\mathbf{n}$ to the opposite side of the cone.

When the voltage over the cell decreased, $\mathbf{n}$ relaxed towards a more planar position, leading to an increased effective optical tilt. Since there was essentially no twist present this time, the sample yielded the maximum optical transmission, as reflected in the second peak (2b) occurring at positive driving voltage. On further voltage decrease the transmission decreased as the liquid crystal relaxed towards the zero-field state, and then the same process recommenced in the other direction.

Directly after the first transmission maxima, 1a and 2a, the current response exhibited the small polarization peak, which we can now attribute to the switching of $\mathbf{n}_{C I}$. The wrong sign of the peak-reducing the current instead of adding to it-is because the $\mathbf{n}_{C I}$ switching occurred at such a high field strength that the dielectric coupling dominated over the polar coupling. When $\mathbf{n}_{C I}$ switched, the twist was expelled and, even though the turning over of $\mathbf{P}$ at the chevron interface corresponded to a small positive charge flow, the director in the major volume of the cell reoriented in such a way that the change in $\mathbf{P}$ direction decreased the current. From the texture we know that, although most parts of the sample switched, the $55 \mathrm{~V}_{p p}$ voltage was high enough to produce some nonswitching domains. Because the domains responded so weakly to the field, their electro-optic response was seen mainly as a background in the switching curves. It is likely that one of the domain types was slightly more common than the other and this may be the reason for the asymmetry in the optical response between positive and negative slope of the driving waveform.

The present study is interesting in the context of recent discussions on polar switching in putatively racemic or achiral smectic- $C$ materials, see e.g., the reports of Walba $e t$ al. [18] and Hird et al. [19]. Such a phenomenon is forbidden by symmetry and therefore suggests a deviation from a perfect 1:1 ratio of $(S)$ - and $(R)$-enantiomers in the case of racemates, or the presence of small amounts of chiral impurities in the case of achiral smectic- $C$ materials. In the sample studied by Walba et al. the former explanation was proven to be the case. As we have shown in this paper, the balance between dielectric and polar switching in $\mathrm{Sm}-C / \mathrm{Sm}-C^{*}$ is extremely sensitive to chiral influence. It is a much more sensitive detector of chirality than the helical superstructure, of which there was no visible trace in the $\mathrm{Sm}-C^{*}$ phase in our mixture. We therefore propose that the discussed unexpected observations of polar switching have their origin in 
the systems not being perfectly racemic/achiral, leading to a very small spontaneous polarization in the smectic- $C$ phase, and that the switching mechanism is of the same type as seen in the chirally doped host 8055 sample.

\section{APPLICATION POTENTIAL}

Perhaps the most important result of this study on an applicational level is the demonstration that a very small amount of a chiral substance, although it is a poor $\mathrm{Sm}-\mathrm{C}^{*}$ dopant (poor in terms of its ability to induce a spontaneous polarization), is enough to radically change the electro-optic response of a Sm- $C$ host liquid crystal. In contrast to the pure host, the doped mixture exhibited a distinct and thresholdless linear response to electric fields. Thus, Sm- $C$ liquid crystals can obviously be used for detecting, and perhaps also quantifying, the presence of chiral substances.

A large part of the paper has been devoted to understanding the complex electro-optic switching mechanism of the doped mixture. This is because it has many attractive properties for use in electro-optic devices such as displays. It features a modulation of the optic axis direction with a range of $\sim 42^{\circ}$ (for this mixture), the texture being uniform in all states thus giving a good contrast [cf. Figs. 8(a) and 8(b)]. The modulation is analog, making gray scale generation easy. The small value of $\mathbf{P}$ is an advantage in the sense that it places small demands on the driving electronics. Although it is not a true in-plane switching mechanism, the variations of director tilt with respect to the cell plane are considerably smaller than in a twisted nematic display. The viewing angle problems should thus be smaller. Furthermore, the switching mechanism utilizes the chevron geometry, a geometry which in most applications of ferroelectric and antiferroelectric liquid crystals is problematic but hard to avoid. It would work well also with uniformly tilted smectic layers.

The asymmetry in the electro-optic response, with plus and minus voltage states exhibiting different twists and different relaxation times, is a potential problem. However, the sample studied in this work was in no way optimized for the mechanism. By tailoring the liquid crystalline properties and cell manufacturing process, it may be possible to diminish the asymmetry to acceptable levels. At the same time, the switching time (at present 1-5 ms) can certainly also be pushed down by optimizing surface anchoring, polarization, and dielectric anisotropy. Since the switching is polarly directed the switching can be made quasiactive even towards the zero-field state by utilizing a smart driving waveform.

For most devices, the mechanism should be used in the low-voltage regime, where the relaxation is reasonably fast. On the other hand, for displays which rarely need to be updated, such as on digital price labels or public information displays, the high voltage regime may actually be attractive, since each gray level can be held in place with a square ac field, making dc-compensation trivial. The domain formation can easily be avoided by first applying a dc pulse at a level above $E_{3}$ (Fig. 6) and then turning to square wave ac driving.

\section{CONCLUSIONS}

We have shown that doping a nonchiral Sm- $C$ liquid crystal host that has positive dielectric anisotropy and weak dielectric biaxiality with a small amount of a nonliquid crystalline chiral substance can produce radical changes in electro-optic switching behavior. Whereas the nonchiral material switched via a Frederiks transition at a distinct threshold, the chirally doped mixture gave a uniform analog response to any electric field. A combination of chevron geometry and small director pretilt at the sample surfaces assured a uniform zero-field state in the doped as well as the undoped material. While the uniformity was intact on application of dc fields of any strength in the chiral sample, the nonchiral sample texture broke up into domains, the boundaries set by the orientation distribution of the director at the chevron interface, at fields above the Frederiks threshold. In both cases, the dielectric coupling to the field dominated the switching process at strong fields but in the chiral sample the polar coupling drove the switching at weak fields, thus selecting a single direction in which the director reoriented across the whole sample area. When driven with ac fields, also the texture of the chirally doped sample broke up into domains if the voltage was above the level which gave maximum electro-optic response, a result of the relaxation from the end states induced by the dielectric coupling being too slow.

The switching mechanism of the chiral mixture is in many respects attractive for application in display devices, since it provides fast thresholdless analog electro-optic response with high contrast, and works with low-polarization materials in a chevron geometry, a structure which in FLC electrooptic devices usually gives rise to problems. The difference in switching behavior compared to the achiral host material shows that the Sm- $C$ liquid crystal phase is extremely sensitive to the effects of chirality, even if the chirality is introduced by a material which is nonliquid crystalline and which induces very small polarization in the resulting $\mathrm{Sm}-C^{*}$ mixture. Also since it has been shown that low concentrations of other small nonliquid crystalline chiral molecules [3] can be detected in achiral smectic- $C$ hosts, electro-optics in systems such as this may provide a basis for detection of chirality.

\section{ACKNOWLEDGMENTS}

The authors thank M. Wand for supplying the 8055 host, L. Wang for help in purifying the chiral dopant, K. D'havé for help in constructing the sample cells and R. Shao, E. Körblova, S. Lagerwall, and F. Giesselmann for helpful discussions. One of the authors (J.L.) gratefully acknowledges financial support from the Swedish Research Council. 
[1] R. B. Meyer, L. Liebert, L. Strzelecki, and P. Keller, J. Phys. (France) Lett. 36, L69 (1975).

[2] H. Stegemeyer, R. Meister, U. Hoffmann, and W. Kuczynski, Liq. Cryst. 10, 295 (1991).

[3] W. Kuczynski and H. Stegemeyer, Chem. Phys. Lett. 70, 123 (1980).

[4] K. Camara, G. Pelzl, and P. Schiller, Cryst. Res. Technol. 31, 131 (1996).

[5] G. Pelzl, P. Schiller, and D. Demus, Liq. Cryst. 2, 131 (1987).

[6] I. Dierking, F. Giesselmann, P. Zugenmaier, G. Pelzl, and P. Schiller, Cryst. Res. Technol. 27, 727 (1992).

[7] G. Pelzl, P. Kolbe, U. Preukschas, S. Diele, and D. Demus, Mol. Cryst. Liq. Cryst. 53, 167 (1979).

[8] N. A. Clark and S. T. Lagerwall, Appl. Phys. Lett. 36, 899 (1980).

[9] S. Inui, N. Iimura, T. Suzuki, H. Iwane, K. Miyachi, Y. Takanishi, and A. Fukuda, J. Mater. Chem. 6, 671 (1996).

[10] B. Park, S. S. Seomun, M. Nakata, M. Takahashi, Y. Takanishi, K. Ishikawa, and H. Takezoe, Jpn. J. Appl. Phys., Part 1 38,
1474 (1999).

[11] P. Rudquist et al., J. Mater. Chem. 9, 1257 (1999).

[12] N. A. Clark, D. Coleman, and J. E. Maclennan, Liq. Cryst. 27, 985 (2000).

[13] N. A. Clark and T. P. Rieker, Phys. Rev. A 37, 1053 (1988).

[14] S. Jenkins, J. Jones, P. Dunn, and R. Richardson, Ferroelectrics 244, 383 (2000).

[15] S. T. Lagerwall, Ferroelectric and Antiferroelectric Liquid Crystals (Wiley-VCH, New York, 1999).

[16] J. Xue and N. Clark, Phys. Rev. Lett. 64, 307 (1990).

[17] I. Musevic, R. Blinc, and B. Zeks, The Physics of Ferroelectric and Antiferroelectric Liquid Crystals (World Scientific, Singapore, 2000).

[18] D.M. Walba, D.J. Dyer, J.A. Rego, J. Niessink-Trotter, R. Shao, and N.A. Clark (unpublished).

[19] M. Hird, J.W. Goodby, N. Gough, and K.J. Toyne, J. Mater. Chem. 11, 2732 (2001). 